\title{
An Auction-Bidding Protocol for Distributed Bit Allocation in RSSI-based Localization Networks
}

\author{
Ahmad A. Ababneh \\ Electrical Engineering Dept., \\ Jordan University of Science \& Technology \\ Irbid, Jordan
}

\begin{abstract}
Several factors (e.g., target energy, sensor density) affect estimation error at a point of interest in sensor networks. One of these factors is the number of allocated bits to sensors that cover the point of interest when quantization is employed. In this paper, we investigate bit allocation in such networks such that estimation error requirements at multiple points of interest are satisfied as best as possible. To solve this nonlinear integer programming problem, we propose an iterative distributed auctionbidding protocol. Starting with some initial bit distribution, a network is divided into a a number of clusters each with its own auction. Each cluster head $(\mathrm{CH})$ acts as an auctioneer and divides sensors into buyers or sellers of bits (i.e., commodity). With limited messaging, CHs redistribute bits among sensors, each bit at a time such that the difference between achieved and required estimation errors within each cluster is reduced in each round. We propose two bit-pricing schemes used by sensors to decide on exchanging bits. Finally, simulation results show that our proposed 'distributed' protocol's error performance can be within $5 \%-10 \%$ of that of a 'centralized' genetic algorithm (GA) solution.
\end{abstract}

Keywords-Target localization; Auction-bidding

\section{INTRODUCTION}

A Distributed sensor network (DSN) consists of a large number of sensors deployed in a region of interest (ROI) with the main task of monitoring certain phenomenon in the ROI [1], [2]. With their ability to continually monitor in harsh and hostile environments with limited human intervention, DSNs bridge the gap between the physical world and our computational world. DSNs have found uses in many fields such as; environmental, industrial, agriculture and defense. However, in many cases it is not sufficient to monitor the phenomenon (e.g., fire) and detect its presence. It might be also necessary to identify the coordinates of the source of the phenomena (i.e., target localization) in order to take meaningful action (e.g., fire control) [3]-[6].

Target localization in general is a nonlinear estimation problem [7]-[9], in which sensors send their nosy data to a fusion center that employs some estimator (e.g., ML estimator) to determine location information. The problem's nonlinearity arises from the nonlinear relationship between sensor measurements (e.g., received signal strength (RSSI) and time difference of arrival (TDOA) ) and target location. RSSI measurements are commonly used due to the simplicity of obtaining them in comparison to other types.
In RSSI-based localization, error performance is dependent on several factors. These include; sensor positions with respect to target, target-related parameters (e.g., energy profile), measurement model [10], [11]. Moreover, in practical networks with imposed bandwidth and energy limits, measurement quantization is usually employed. In such a case, the localization error performance further depends on both the number of bits allocated to each sensor and the quantization thresholds used [12].

Due to the complicated relationship between the above mentioned parameters and the error performance, it becomes important to devise intelligent methods for bit allocation in RSSI networks. Furthermore, and due to the large number of sensors typically found in DSN, it is important for bit allocation to be scalable and easy to implement.

We state the problem we study in this paper as follows; Given a network of $M$ sensors deployed in an ROI that contains multiple points of interest with corresponding estimation error requirements and having some given initial bit allocation distribution, how can we re-allocate these bits in a "distributed" fashion such that error requirements at the points of interest are met as best as possible?. To solve this problem, propose a novel distributed bidding/auction protocol for bit re-allocation. Next, we provide an overview of some related works to the bit allocation problem in RSSI-based localization networks.

In [13], an iterative two-stage algorithm for bit allocation and threshold selection is proposed with the goal of minimizing the average overall error. The first stage deals with the reconstruction of the quantized sensor measurement at the fusion center (FC). The second part is concerned with the error between the actual location of the target and its estimated location. We note that the proposed algorithm constructs the quantizer using a training data set according to some given probability distribution of the target's possible location. In addition, the authors propose a simple equal distance divided quantizer (EDQ) for threshold selection, where each quantization interval corresponds to a quantization ring within the sensor field.

More recently, in [14] and [15], the authors propose several bit allocation methods for the target tracking problem with the goal of minimizing estimation error. As a cost function, the authors use the determinant of the Fisher information matrix (FIM). To solve this problem, the authors propose an 'approximate' recursive dynamic programming (A-DP) 
approach for bit allocation. In addition, a modified version of the Breiman, Friedman, Olshen, and Stone (GBFOS) algorithm is proposed. Both algorithms are shown to have a comparable performance. In [16], the authors devise several bit allocation algorithms to meet error requirements at multiple points within the region of interest rather than a single point which enables the considerations of multiple subregions with different levels of importance. Furthermore, an adaptive thresholding scheme is devised in [16] that incorporates error requirements within a sensor's sensing zone in threshold selection.

We note the algorithms presented in the works above are 'centralized' in nature, that require sensors to send relevant data to a central processor to perform allocation. This centralized approach might not be computationally practical for dense networks with a large number of sensors. In addition, it might result in inadequate performance, especially if requirements are rapidly changing. In contrast, the algorithm we propose in this paper is distributed in nature and requires information exchange between a sensor and the sensors in its immediate neighborhood. The second difference is the fact that in most of these works, error minimization was considered at a single point. This is in contrast to this work, in which the goal is to meet error requirements over an area of interest.

The bit allocation algorithm we propose in this paper is an auction/bidding-type algorithm. As a network become more complicated and the operation of its different components (e.g., sensors) more inter-related, its management becomes more difficult. One important problem that faces such a DSN is resource allocation [17]. One method for solving this problem is auction theory, which has been applied to bandwidth, power and time-slot allocation problems [18]-[20] in communication networks. Auction theory has been applied as well to sensors networks. For example, the authors in [21] propose a bidding protocol for mobile sensors deployment with the goal of maximizing the coverage within a region of interest. In this protocol, static sensors detect coverage holes and estimate their size and send this information to near-by mobile sensors which only relocate to the new location if the improvement in coverage after movement is larger than coverage degradation at the original sensor location. The proposed coverage-maximizing protocol is distributed and is scalable to dense networks.

In this paper, we consider an (ROI) with $N$ points of interest. Each point is assigned a minimum estimation requirement that is to be met. A number $M$ of sensors are deployed, each with an initial number of quantization bits that need not be uniform. It is of interest to reallocate bits such that specified estimation error requirements are met as best as possible. To solve this problem, we propose a novel iterative auction/bidding protocol for bit reallocation. In each iteration/round, the network is divided into clusters, each with its own cluster head $(\mathrm{CH})$. The $\mathrm{CH}$ acts as an auctioneer in an auction. The $\mathrm{CH}$ then divides sensors in its cluster to either buyers or sellers where the commodity to be exchanged is bits. The pricing policy (i.e., valuation) of bits can be specified in several ways. Here, we propose two valuation policies in terms of two factors; the number of bits that a sensor already uses and the difference between achieved and required estimation requirements within a sensor's sensing radius. The $\mathrm{CH}$ deallocates a bit from a seller sensor with the lowest valuation, to the buyer with the highest valuation.
Simulation results show that the proposed distributed algorithm can provide a comparable performance to that of a centralized genetic algorithm (GA) solution.

The paper is organized as follows; in Section (II) we discuss the system model and our problem set-up. In Section (III), we introduce the proposed bit allocation methods. Simulation results are discussed in Section (IV) and conclusions are summarized in Section (V).

\section{SyStem MOdEL AND PROBLEM Formulation}

We consider an 2-D region of interest (ROI) of dimensions $b \times b$ with $N$ points of interest in the ROI. The coordinates of these points are given as $\left\{\mathbf{p}_{n}=\right.$ $\left.\left[x_{n}, y_{n}\right]^{\prime}, n=1,2, \ldots, N\right\}$. Minimum estimation error requirements $\left\{\mathbf{E}_{\mathrm{req}}(n), n=1,2, \ldots, N\right\}$ are specified for each point. Using $\mathbf{E}_{\text {req }}$ enables us to divide the ROI into different subregions with varying levels of importance if needed.

The network consists of $M$ static sensors with known positions denoted as $\left\{\mathbf{s}_{m}=\left[x_{m}, y_{m}\right]^{\prime}, m=1,2, \ldots, M\right\}$. Sensors are assumed to provide received signal strength indicator (RSSI) measurements which are easier to obtain than other measurement types (e.g.,time of arrival (ToA)) [22], [23].

Let $\mathbf{p}_{t}=\left[x_{t}, y_{t}\right]^{\prime}$ denote the target's position, then the RSSI measurement of the $m$-th sensor is given as [24], [25]

$$
z_{m}=\sqrt{P_{0}} e^{\frac{-\alpha}{2} d_{t, m}^{2}}+w_{m}, \quad m=1,2, . . M
$$

where, $P_{0}$ denotes the energy emitted by the target and $d_{t, m}$ denotes the distance between the $m$-th sensor and $\mathbf{p}_{t}$. The parameter $\alpha$ is a decay factor. The noise $w_{m}$ is assumed to be i.i.d zero-mean Gaussian $\left(w_{m} \sim \mathcal{N}\left(0, \sigma^{2}\right)\right)$.

To reduce energy and bandwidth usage, sensors are assumed to transmit their measurements quantized. Let $R_{m}$ denote the number of bits used by the $m$-th sensor and let $\left\{\eta_{m}^{l}, l=1,2, \ldots, 2^{R_{m}}-1\right\}$ denote its quantization thresholds, then the quantized measurement of the $m$-th sensor which is denoted as $z_{m}^{\mathrm{q}}$ is mapped as follows

$$
z_{m}^{\mathrm{q}}= \begin{cases}0 & , \text { if }-\infty<z_{m} \leq \eta_{m}^{1} \\ 1 & , \text { if } \eta_{m}^{1}<z_{m} \leq \eta_{m}^{2} \\ \cdot & \\ 2^{R_{m}}-1 & , \text { if } \eta_{m}^{2^{R_{m}}-1}<z_{m} \leq \infty\end{cases}
$$

Let $\mathbf{E}(n)$ denote the achieved estimation error at the $n$-th point, then $\mathbf{E}(n)$ quantifies how accurate is the estimator in estimating the location of the target if it were placed at the $n$-th point. The mean square error $\mathbf{E}(n)$ is defined as

$$
\mathbf{E}(n)=\mathbf{E}\left[\left(\mathbf{p}_{n}-\hat{\mathbf{p}_{n}}\right)\left(\mathbf{p}_{n}-\hat{\mathbf{p}_{n}}\right)^{\mathrm{T}}\right]
$$

where, $\mathrm{E}[\cdot]$ denotes the expectation operator.

The error $\mathbf{E}(n)$ can be bounded using the Cramer-Rao lower bound(CRLB) [26], [27]. In many cases (e.g., large SNR measurements, dense network), the CRLB can be used as an approximation of the actual estimation error.

In our problem, $\mathbf{E}(n)$ can be bounded using the CRLB as

$$
\mathbf{E}(n) \geq \text { Trace }\left[\left(\mathbf{J}_{n}\right)^{-1}\right]
$$


where, $\mathbf{J}_{n}$ is the Fisher information matrix (FIM) corresponding to the $n$-th point. For the derivation of the FIM matrix for the quantized RSSI measurement model, the reader is referred to [24] and is given as

$$
\mathbf{J}=\left[\begin{array}{ll}
J_{11} & J_{12} \\
J_{21} & J_{22}
\end{array}\right]
$$

where, the FIM elements are given as [24], [25]

$$
\begin{aligned}
J_{11} & =\sum_{m} \beta_{m}\left(x_{n}-x_{m}\right)^{2} \\
J_{12} & =J_{21}=\sum_{m} \beta_{m}\left(x_{n}-x_{m}\right)\left(y_{n}-y_{m}\right) \\
J_{22} & =\sum_{m} \beta_{m}\left(y_{n}-y_{m}\right)^{2} .
\end{aligned}
$$

The parameter $\beta_{m}$ is given as

$$
\beta_{m}=\frac{\alpha^{2} P_{0} e^{-\alpha d_{m, n}^{2}}}{2 \pi \sigma^{2}} \gamma_{m}
$$

where $\lambda_{m}$ and $\gamma_{m}$ are given as

$$
\lambda_{m}=\frac{\alpha^{2} P_{0} e^{-\alpha d_{m, n}^{2}}}{2 \pi \sigma^{2}}
$$

and

$$
\gamma_{m}=\sum_{l} \frac{1}{p_{m}^{l}}\left[e^{\frac{-\left(\eta_{m}^{l}-a_{m}\right)^{2}}{2 \sigma^{2}}}-e^{\frac{-\left(\eta_{m}^{l+1}-a_{m}\right)^{2}}{2 \sigma^{2}}}\right]^{2}
$$

where, $p_{m}^{l}$ is the probability that the quantized measurement $z_{m}^{\mathrm{q}}$ takes on the value $l$ and is given as

$$
\begin{aligned}
p_{m}^{l} & =\operatorname{Pr}\left(z_{m}^{\mathrm{q}}=l\right) \\
& =Q\left(\frac{\eta_{m}^{l}-a_{m}}{\sigma}\right)-Q\left(\frac{\eta_{m}^{l+1}-a_{m}}{\sigma}\right) .
\end{aligned}
$$

Based on the equations above (Eqns.(6)-(11)), that the estimation error depends on several parameters (e.g., $P_{0}, \alpha$ and $\sigma^{2}$ ). We also note that a sensor's contribution to reduction of error at a point is inversely proportional with the distance separating it from the point of interest. Finally, we note that the number of quantization bits and thresholds are incorporated in the $\gamma_{m}$ parameter in Eqn. (11). In general, the more bits allocated combined with an intelligent choice of quantization intervals results in increase of the sensor's contribution to estimation error reduction.

Thus, it is evident that the distribution of bits within the ROI is an important factor in determining the overall error performance of the network. An informed allocation of bits is especially important for networks deployed in areas with different estimation requirements. It is also critical in the case of varying requirements or network topology (e.g., death or malfunction of sensors) in which loss of bits needs to be compensated.

The problem we study in this paper can be stated mathe- matically as follows

$$
\begin{array}{ll}
\underset{\mathbf{R}}{\operatorname{minimize}} & \sum_{n=1}^{N}\left(\mathbf{E}_{\text {req }}(n)-\mathbf{E}(n)\right)^{2} \\
\text { subject to } & \mathbf{R} \succeq \mathbf{0}, \\
& \sum_{m=1}^{M} R_{m}=R_{T},
\end{array}
$$

where, $\mathbf{R}=\left[R_{1}, R_{2}, \ldots, R_{m}\right]$ is the vector of the number of bits allocated to each sensor and $R_{T}$ is the total number of bits.

The allocation problem in Eqn. (14) is an integer nonlinear programming problem. Because of its computational complexity, especially for dense networks, it is desirable to develop computationally tractable algorithms for solving such a problem.

One important note is the fact that the estimation error achieved at some points might not be defined. Localization in 2-D setups requires that a point be covered (i.e., within the sensing radius of a sensor) with at least 3 sensors. Hence, if a point is covered by less than 3 sensors, then regardless of how many bits are allocated to the sensors covering it there will be no meaningful achieved estimation error. We denote this as having $\mathbf{E}(n)=\infty$. To alleviate this problem, we propose the following logarithmic error definition [16]

$$
\mathbf{E}^{L}(n)=\log \left(1+\frac{1}{\mathbf{E}(n)}\right)
$$

which solves the problem of dealing with errors. Hence, we can consider modifying the objective function in Eqn. 14 to be in terms of the logarithmic errors.

We next present a distributed protocol for solving the above mentioned allocation problem.

\section{Proposed Allocation Protocol}

The bit allocation problem in RSSI localization is difficult due to the nonlinearity between achieved estimation error and the number of bits allocated to a sensor. This is in addition to the error dependence on the actual quantization thresholds used. Another factor is the dependence of estimation error at one point on not only on a single sensor, but on the total number of sensors covering this point along with their quantization bits and thresholds. We later refer to this dependence as the coupling problem.

To solve this problem we next outline our proposed bit allocation protocol.

\section{A. Proposed Protocol}

To establish an auction, we need several elements; sellers, buyers, auctioneer and commodities to be exchanged with certain prices and revenues. To attain these elements, we divide the network into several clusters, each with its own cluster head $(\mathrm{CH})$. Sensors in each cluster participate as either sellers or buyers with the $\mathrm{CH}$ acting as an auctioneer. To reduce communication costs, the $\mathrm{CH}$ (i.e., auctioneer) collects data about possible sellers and buyers in its cluster and makes the allocation decision. Using some bit valuation (i.e., a pricing 
scheme which will be discussed later), a sensor is to deallocate a bit (i.e., sell) to another sensor in its cluster if it produces a better overall network valuation (e.g., error performance) than if bit were to be kept by the sensor. Similarly, a sensor is permitted to use an additional bit (i.e., buy), that has been deallocated from another sensor, if it produces a better overall network valuation. To reduce communication cost between sensors, communication is restricted to the $\mathrm{CH}$. The proposed protocol can be outlined as follows

1) The $\mathrm{CH}$ calculates its own selling price according to the specified scheme and transmits its price to its cluster nodes.

2) Each sensor locally calculates its own selling price and those with a price lower than that of the $\mathrm{CH}$ identify themselves as sellers and inform the $\mathrm{CH}$ of their status and price.

3) Next, the $\mathrm{CH}$ calculates its buying price and transmits it to cluster sensors.

4) Sensors locally calculate their buying price and compare to that of the $\mathrm{CH}$. Sensors with a higher buying price than that of the $\mathrm{CH}$ identify as buyers and similarly send their information to the $\mathrm{CH}$.

5) The $\mathrm{CH}$ then deallocates a bit from the sensor with the lowest selling price and allocates it to the sensor with the highest buying price.

6) Repeat the above steps as much as specified by the user.

In addition to being an auctioneer, the $\mathrm{CH}$ acts as a possible buyer or seller. This is true since it provides a ceiling on the selling price and a floor on the buying price which is useful for limiting unnecessary communication.

Notes:

1) The selection of $\mathrm{CHs}$ can be achieved using a cluster head selection algorithm. A popular approach is using the LEACH algorithm which is employed in this paper [28], [29]. Using this algorithm, a sensor $(s)$ generates a random number between 0 and 1 . If this number is less than a certain threshold $T(s)$, then the sensor becomes a $\mathrm{CH}$ for this round. The threshold $T(s)$ is given as [28], [30]

$$
T(s)= \begin{cases}\frac{p_{\mathrm{CH}}}{1-p_{\mathrm{CH}}\left(r \quad \bmod \frac{1}{p}\right)} & \text { if } s \in G \\ 0 & \text { else }\end{cases}
$$

where, $p_{\mathrm{CH}}, r, G$ represent, respectively, the desired percentage of cluster-heads, the current round number, and the set of nodes that have not been clusterheads in the last $1 / p_{\mathrm{CH}}$ rounds. We note that $p_{\mathrm{CH}}=\frac{C}{M}$, where $C$ is the number of $\mathrm{CH}$ to be selected. Sensors closer to a $\mathrm{CH}$ join the cluster corresponding to this $\mathrm{CH}$.

2) One problem resulting from the coupling of error performance is the collision problem. We define the collision problem in this context as the problem of interfering sensors whose sensing zones (i.e., disc of radius $R_{s}$ ) overlap with the domains of other sensors that fall within another cluster. Under such situation, $\mathrm{CHs}$ of two adjacent clusters might deallocate bits from neighboring sensors concurrently. This might produce a change in estimation errors more than the one reported by sensors to be their selling price, which can not be matched by improvement in estimation error reported by the winning sensor. On the other hand, bits can be allocated concurrently to adjacent sensors from two clusters which causes overmeeting estimation requirements at some area while requirements at other areas are not met.

To solve this problem, it is possible to force the $\mathrm{CHs}$ to be a certain distance away from each other such that sensors that belong to a cluster do not have overlapping domains with sensors that belong to other clusters. If $R_{\mathrm{CH}}$ and $R_{s}$ denote the clustering and sensing radii respectively, then the distance between $\mathrm{CHs}$ $R_{\Delta \text { сн }}$ that eliminates the collision problem should be such that

$$
R_{\Delta \mathrm{CH}} \geq 2\left(R_{\mathrm{CH}}+R_{s}\right)
$$

We note that using a smaller $R_{\Delta \mathrm{cH}}$, does not necessarily mean that collision will always happen as this depends on sensor positions within the clusters. However, it implies that randomly-occurring degradations in estimation performance are possible. One problem with this approach that it sets a bound on the number of clusters (i.e., auctions) that can be formed in each iteration resulting in a slow reduction of estimation errors. Furthermore, it produces varying number of $\mathrm{CHs}$ in each round.

3) The collision problem can also affect bidding in the same cluster, especially when a sensor evaluates bits assuming neighboring sensors are to keep their bits fixed. Assume a sensor submits its selling price and is chosen as a seller. Furthermore, assume the winning sensor's domain overlaps that of the selling sensor. Under this setup, the improvement in estimation error is likely less than what was anticipated by the winning sensor. Thus a lower bidding price might have been more suitable (i.e., another sensor could have won). Different methods can be used to solve the above problem. For example, sensors can calculate their valuations taking into account changes (i.e., increase or decrease) in number of bits at neighboring sensors. However, this comes at the expense of increased computational complexity. Another method, that is employed in this paper, is for each selling sensor to exclude overlapping sensors (i.e., sensors less than $R_{s}$ away) from the pool of its possible buyers.

4) With no constraints on the number of bits that a sensor can have, a sensor might end up selling all its bits. Thus, the sensor will not report any measurements resulting in a coverage loss (i.e., as if the sensor does not exist when calculating actual estimation errors). One possible solution is for a sensor to stop participation in selling when it has one bit left.

\section{B. Bit Valuation}

We propose two possible bit valuation (i.e., pricing schemes) methods that require a limited amount of message passing. These schemes require knowledge of positions of 


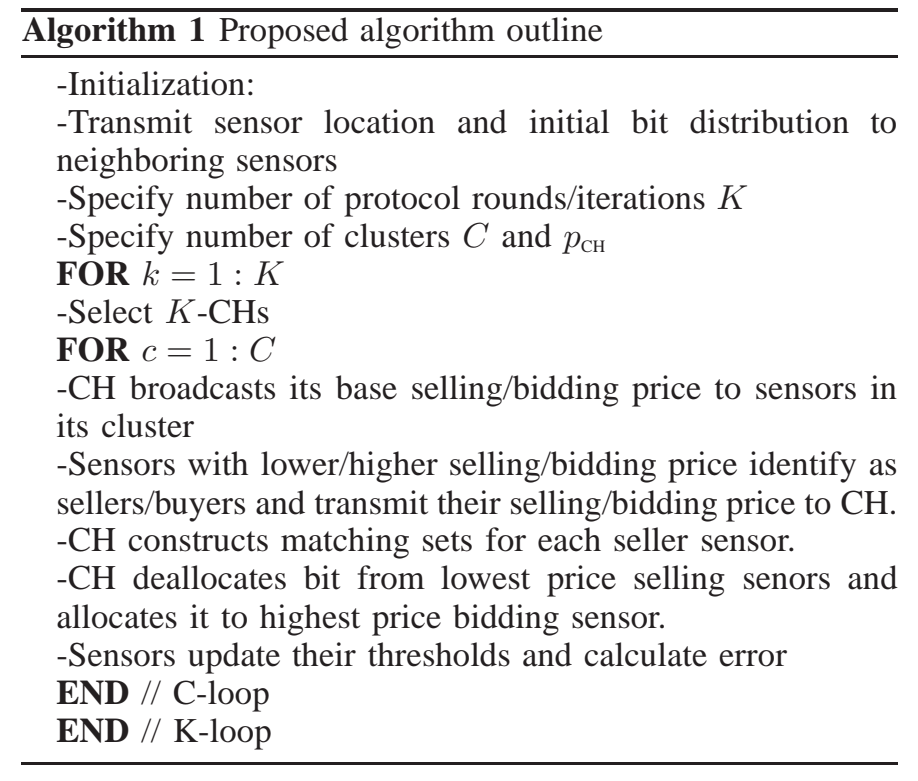

sensors that are less than $R_{s}$ units away which can be performed one time after the initial deployment. Knowledge of the number of bits allocated to each sensor is also required, which can be transmitted by the $\mathrm{CH}$ after each algorithm iteration.

1) Estimation error difference: When a sensor wants to determine its selling price, it locally calculates the degradation in estimation performance when one of its bits is removed. Let $I_{m}$ indicate the set of point indexes within the sensing radius of the $m$-th sensor. Then the total localization error at these points is given as

$$
e(m)=\sum_{i \in I_{m}^{+}} \Delta E^{L}(i)
$$

where, $I_{m}^{+}$denotes indexes of points where requirements are not met and $\Delta E(i)$ is defined as

$$
\Delta E(i)=E_{\text {res }}^{L}(i)-E_{\text {req }}^{L}(i) .
$$

When calculating the selling price, the sensor locally calculates the total localization error $e^{-}(m)$ if one bit was removed. The selling price $p_{m}^{-}$is then defined as

$$
p_{m}^{-}=\left|e^{-}(m)-e(m)\right|
$$

Therefore, as the degradation in anticipated estimation error decreases, the sensor will be more willing to sell its bit. Similarly, the bidding price is then given as

$$
p_{m}^{+}=\left|e^{+}(m)-e(m)\right|
$$

where, $e^{+}(m)$ is the error if one bit was added to the sensor. Thus, the larger the improvement in error performance, the greater is the bidding price $p_{m}^{+}$.

The above scheme is not the only scheme that we can propose. We next, propose a pricing scheme that in addition to estimation error, incorporates the number of used bits in calculating a bit's price.

2) Error-Bit Efficiency: In this pricing scheme, we propose to incorporate the error per bit efficiency metric. This enables us to quantify how efficient is the sensor using each of its bits. Let $R(m)$ denote the number of the $m$-th sensor bits, we define the error-bit efficiency $\eta_{m}$ as

$$
\eta(m)=\frac{\% e(m)}{R(m)}
$$

where,

$$
\% e(m)=\sum_{i \in I_{m}^{+}} \frac{E_{\text {res }}^{L}(i)-E_{\text {req }}^{L}(i)}{E_{\text {req }}^{L}(i)}
$$

Using the efficiency measure, we can now define the selling/bidding price as

$$
p_{m}^{ \pm}=\left|\eta^{ \pm}(m)-\eta(m)\right|
$$

The selling price defined above indicates the relative change in bit efficiency when one bit is to be removed. The smaller the efficiency change, the more willingness to sell the bit and thus the lower price. In case of bidding for bits, sensors with the most efficiency will propose higher bidding prices.

In the next section, we investigate the performance of the proposed protocol.

\section{Simulation Results}

To study the performance of our proposed bidding protocol, we use the average error criterion which we define as the difference between achieved and required estimation error requirements over the whole region of interest at the $k$-th protocol round and is given as

$$
\text { Average } \operatorname{Error}(l)=\sum_{i \in I_{+}} \Delta \mathbf{E}_{k}^{L}(i),
$$

where, $I_{+}$denotes the set of points where error requirements are not satisfied. We also employ the normalized average error criterion which is the overall average error at the $k$-th iteration normalized with respect to the initial average error before any bidding is performed denoted as Average Error $_{\text {initial }}$. That is;

$$
\text { Normalized } \operatorname{Error}(k)=\frac{\text { Average } \operatorname{Error}(k)}{\text { Average } \operatorname{Error}_{\text {initial }}},
$$

We note that we use a number 100 Monte Carlo iteration to average our results over. Another criterion we use is the relative average error gap with respect to the error of that of a solution generated by a 'centralized' genetic algorithm (GA) approach, and is defined as

$$
R E_{\mathrm{GA}}(k)=\frac{\text { Average Error }(k)-\text { Average } \text { Error }_{\mathrm{GA}}}{\text { Average } \text { Error }_{\mathrm{GA}}}
$$

where, Average Error $_{\mathrm{GA}}$ is the error corresponding to the GA solution. We note that in all of our GA simulations we use a population size of 100 with 30 generations. We also note that the EDQ threshold selection method is used in all of our simulations.

In the first experiment, we investigate the behavior of our proposed protocol for different initial energy $P_{0}$ levels for both proposed pricing schemes. We consider a $20 \times 20$ ROI, with uniform estimation requirements set to $1.5 \mathrm{~m}^{2}$. Additional experiment parameters as listed in Table. I below (notably, 


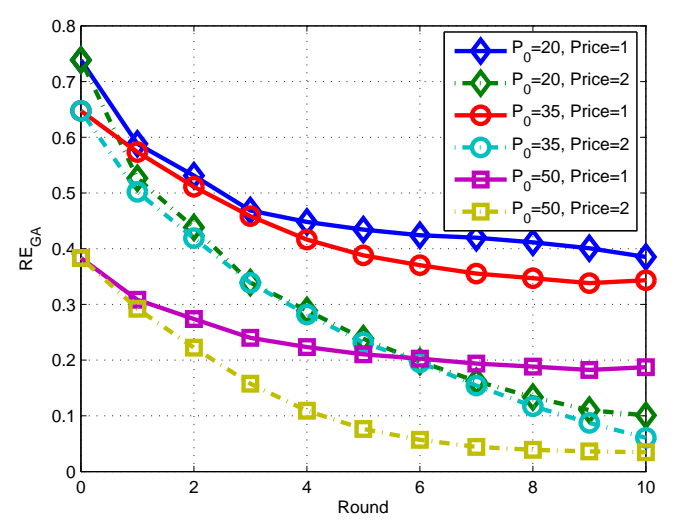

Fig. 1: $R E_{\mathrm{GA}}(k)$ vs. rounds for different $P_{0}$. Price=1 and 2 refer to the error difference and error-bit efficiency schemes, respectively.

$M=30$ sensors with $R_{m}=3$ bits for each sensor in addition to using $C=5 \mathrm{CHs}$ with $K=10$ rounds).

\begin{tabular}{|c|c|}
\hline$b$ & 20 \\
\hline$\sigma^{2}$ & 1 \\
\hline$M$ & 30 \\
\hline$N$ & 200 \\
\hline$R_{s}$ & 5 \\
\hline$\alpha$ & 0.1 \\
\hline$C$ & 5 \\
\hline$L$ & 10 \\
\hline$R_{m}$ & 3 \\
\hline
\end{tabular}

TABLE I: Parameters of Experiment Set 1

The relative error gap $R E_{\mathrm{GA}}$ is depicted in Fig. 1 below. We first note that the $R E_{\mathrm{GA}}(k)$ decreases as more rounds of the protocol are executed and as $P_{0}$ is increased. As $P_{0}$ is increased, sensor measurements become more accurate and sensors can more accommodate selling their bits to other sensors. This is especially true for sensors that are within an area of high coverage (i.e., large number of overlapping sensors) where requirements are more satisfied relative to other low coverage areas (i.e., with fewer sensors overlapping) where requirements are less satisfied. More notably, the use of the second pricing scheme (i.e., error-bit efficiency) results in a smaller gap than that of the first pricing scheme (error difference). For example, using the error difference scheme results in an error gap of between $20 \%-38 \%$. However, a smaller gap of between $5 \%-10 \%$ can be achieved using the second scheme after $K=10$ rounds. This is a direct result of the incorporation of the relative error difference as well as the number of bits assigned to a sensor.

The second experiment investigates the error performance as we vary the number of cluster heads $(C)$. The experiment parameters are similar to that of the previous experiment with $P_{0}=35$ and a variable $C$. Results are depicted in Fig. 2 below. We note that increasing $C$ increases the error reduction. This is expected as having more clusters implies more auctions are being performed which results in a faster reduction of errors.
However, we note that increasing $C$ has a diminishing return behavior, especially when using the second pricing scheme where increasing $C$ from 3 to 5 results in a small overall improvement in error reduction.

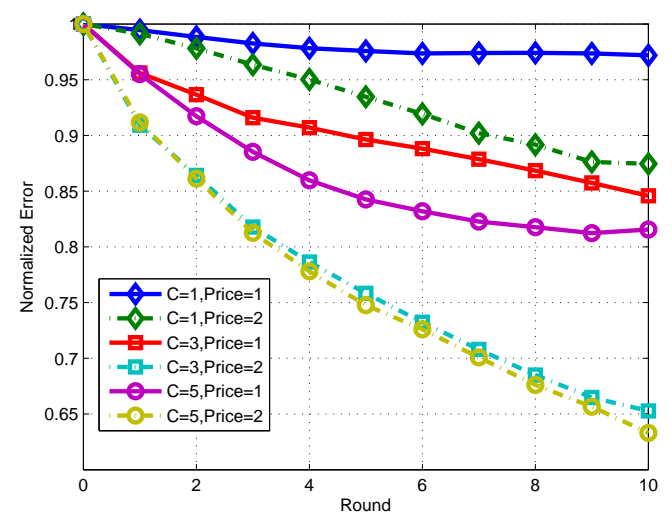

Fig. 2: Normalized error vs. rounds for different $C \mathrm{CHs}$

We next consider a different setup in which sensors are uniformly deployed but with nonuniform bit allocation. In particular, sensors in the upper right corner of the ROI are assigned a number of bits (which we call 'corner bits') different from other sensors which are each assigned one bit $\left(R_{m}=1\right)$. It is evident (see Fig. 3) that the proposed protocol is capable of reducing the average error even when possible bits to be traded are concentrated in one area. We remind the reader that sensors with one bit are prohibited from selling in our protocol. Thus, the more bits are available for exchange, the better the protocol reduces error using any of the pricing schemes. This is especially true fo the error-bit efficiency price which produces a lower selling/bidding price when the number of allocated bits is larger than those of other sensors in cluster. It is noted that this nonuniform bit distribution amplifies the performance difference between the two proposed pricing schemes. An example of the re-distribution of bits among sensors is shown in Figs. 4, 5 that show the initial distribution of bits (with 4 corner bits) as well as that of the GA algorithm's and the proposed protocol pricing schemes.

In the fourth experiment, we consider an ROI with nonuniform estimation requirements as shown in Fig. ?? and parameters as shown in Table. I . From Fig. 7, we note that the more stringent requirements become (i.e., lowering $E_{\text {req }}$ ), the larger is the error gap with respect to the optimal solution. This is especially true before the protocol is implemented. Satisfying stringent requirements requires moving bits closer to the required region while not degrade performance at other regions. We further note, the as requirements become less stringent (i.e., from $E_{\text {req }}=0.5$ to $1.5 \mathrm{~m}^{2}$ ) the larger is the error reduction. That is because estimation errors are more satisfied and thus bits can be traded and moved to areas with more stringent requirements.

Figs. 8 and 9 show an example of the error difference distribution over the ROI, with $E_{\text {req }}=0.5$ set to the lower left corner. The darker the areas (i.e., lower vale on the color bar) the more the error is satisfied. 


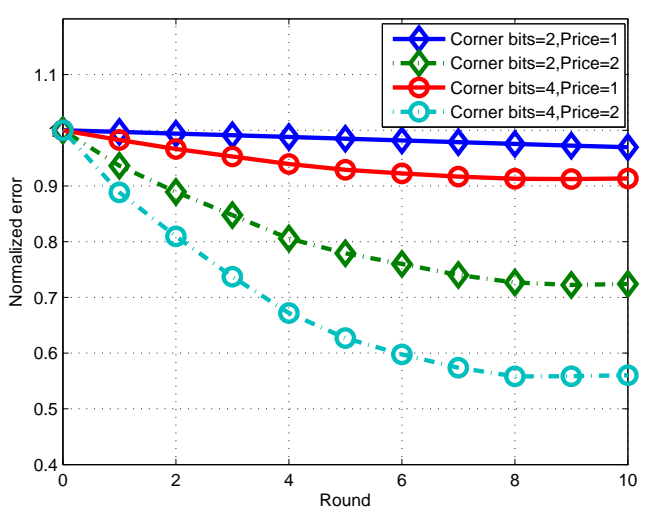

Fig. 3: Normalized error vs. rounds for different bit distributions

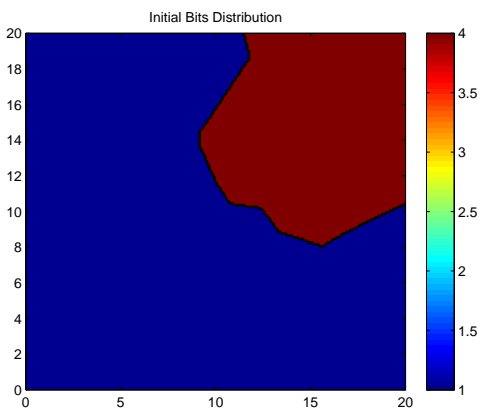

(a) Initial

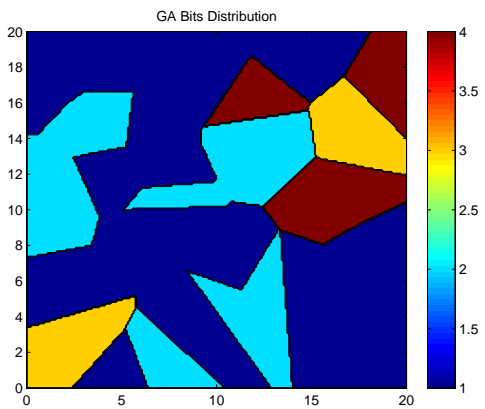

(b) GA

Fig. 4: Initial and GA Bit distribution

\section{CONCLUSION}

In this paper, we studied the bit allocation problem for the RSSI-based localization network. Having an initial distribution of sensors, the goal is to re-allocate bits such that estimation requirements at multiple points in the ROI are satisfied as best as possible. To solve this problem, a novel iterative distributed auction/ bidding protocol is introduced. The network is divided into clusters, with each cluster head acting as an auctioneer and dividing sensors into buyers or sellers of bits. Two pricing schemes are proposed; the error difference and error

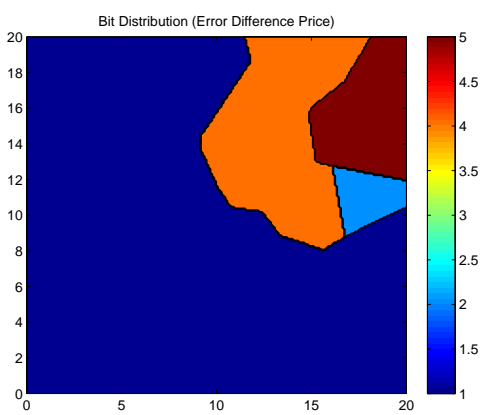

(a) Error difference price

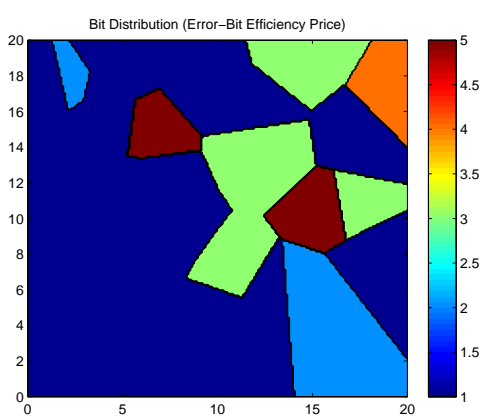

(b) Error-Bit efficiency price

Fig. 5: Bit distribution using proposed protocol

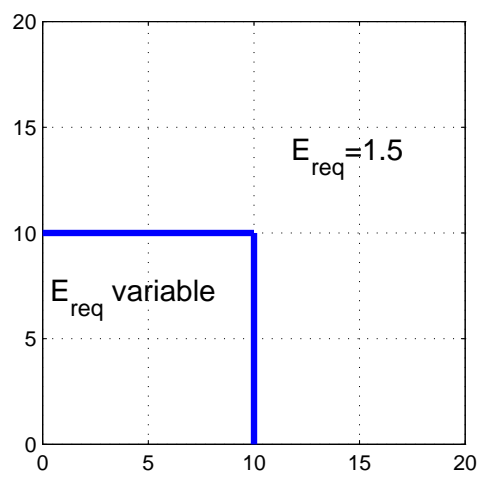

Fig. 6: Nonuniform estimation requirements

difference-bit efficiency schemes. Simulation results indicate that the proposed protocol can achieve an error performance that is within less than $10 \%$ of that a centralized genetic algorithm (GA) approach.

\section{REFERENCES}

[1] I. F. Akyildiz, W. Su, Y. Sankarasubramaniam, and E. Cayirci, "Wireless sensor networks: a survey," Computer Networks, vol. 38, no. 4, pp. 393422, 2002.

[2] J. Yick, B. Mukherjee, and D. Ghosal, "Wireless sensor network survey," Computer Networks, vol. 52, no. 12, pp. 2292-2330, 2008. 


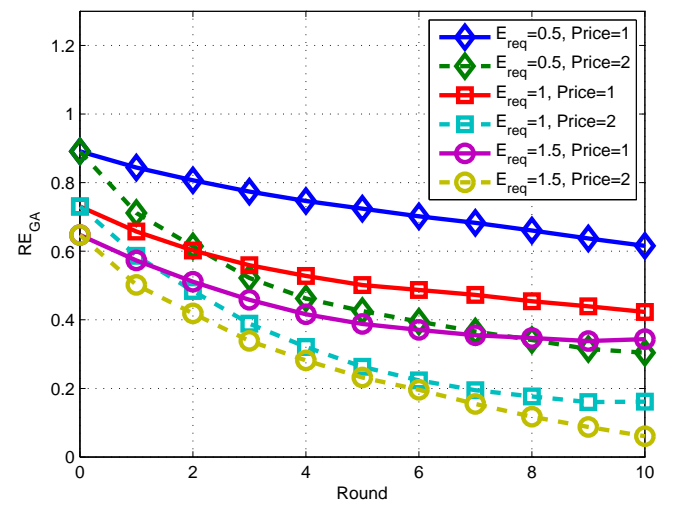

Fig. 7: $R E_{\mathrm{GA}}(k)$ vs. rounds for different requirements

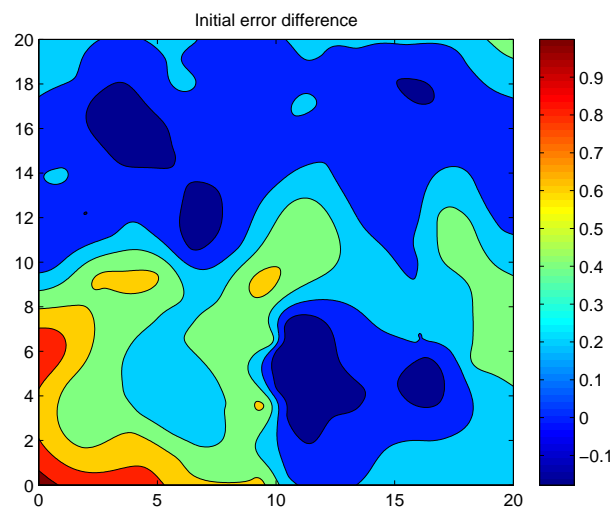

(a) Initial

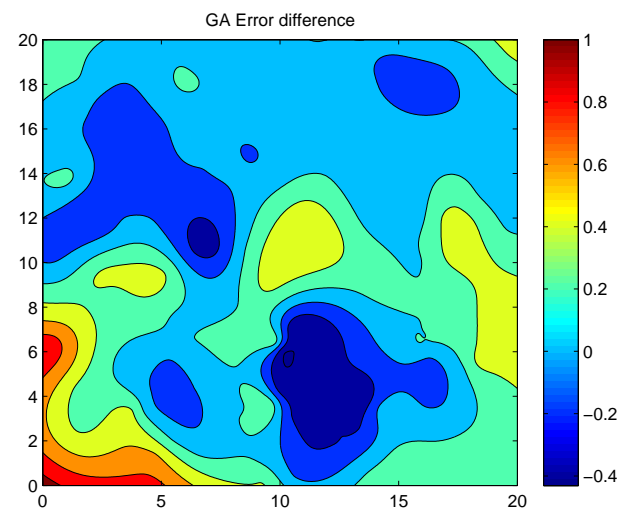

(b) GA

Fig. 8: Initial and GA error difference distribution

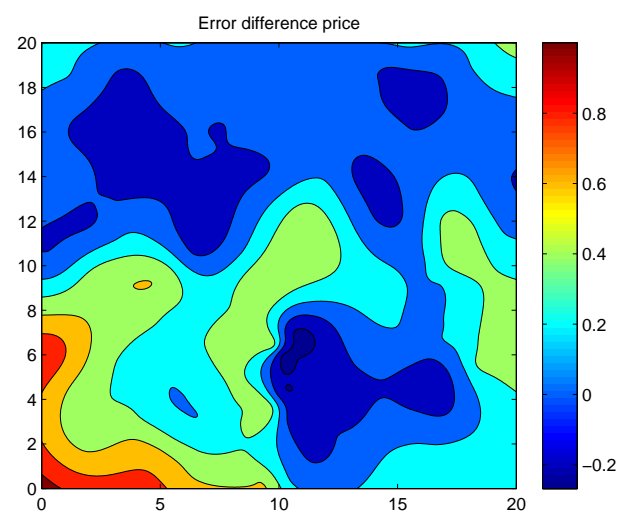

(a) Error difference price

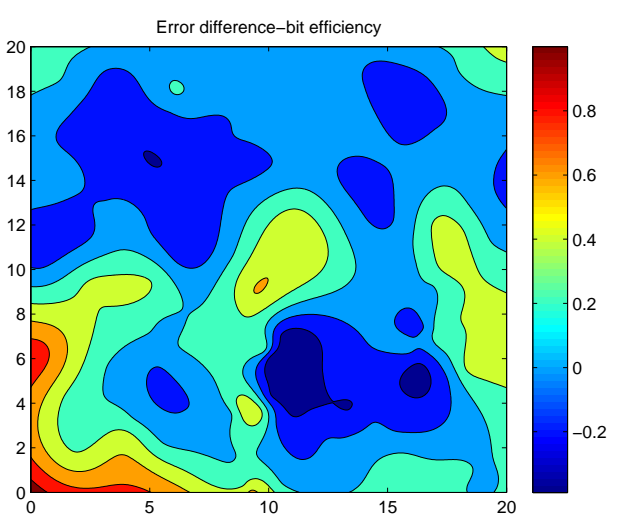

(b) Error-Bit efficiency price

Fig. 9: Error distribution using proposed protocol

[3] H. Karl and A. Willig, Protocols and architectures for wireless sensor networks. Wiley, 2007.

[4] G. Mao, B. Fidan, G. Mao, and B. Fidan, Localization Algorithms and Strategies for Wireless Sensor Networks. Hershey, PA: Information Science Reference - Imprint of: IGI Publishing, 2009.

[5] P. U. Lima, "A bayesian approach to sensor fusion in autonomous sensor and robot networks," IEEE Instrumentation Measurement Magazine, vol. 10, no. 3, pp. 22-27, June 2007.

[6] D. Pizarro, M. Mazo, E. Santiso, M. Marron, and I. Fernandez, "Localization and geometric reconstruction of mobile robots using a camera ring," IEEE Transactions on Instrumentation and Measurement, vol. 58, no. 8, pp. 2396-2409, Aug 2009.

[7] X. Sheng and Y.-H. Hu, "Maximum likelihood multiple-source localization using acoustic energy measurements with wireless sensor networks," Signal Processing, IEEE Transactions on, vol. 53, no. 1, pp. 44-53, 2005.

[8] G. Mao, B. Fidan, and B. Anderson, "Wireless sensor network localization techniques," Computer Networks, vol. 51, no. 10, pp. 2529-2553, 2007.

[9] V. K. Chaurasiya, N. Jain, and G. C. Nandi, "A novel distance estimation approach for 3d localization in wireless sensor network using multi dimensional scaling," Information Fusion, vol. 15, pp. 5-18, 2014.

[10] A. N. Bishop, B. Fidan, B. D. Anderson, P. N. Pathirana, and K. Dogançay, "Optimality analysis of sensor-target geometries in passive localization: Part 2-time-of-arrival based localization," in Proceedings of the 3rd International Conference on Intelligent Sensors, Sensor Networks and Information Processing (ISSNIP), 2007. 
[11] A. N. Bishop and P. Jensfelt, "An optimality analysis of sensortarget geometries for signal strength based localization," in Intelligent Sensors, Sensor Networks and Information Processing (ISSNIP), 2009 5th International Conference on. IEEE, 2009, pp. 127-132.

[12] A. Gersho and R. M. Gray, Vector quantization and signal compression. Springer, 1992.

[13] Y. H. Kim and A. Ortega, "Quantizer design for energy-based source localization in sensor networks," Signal Processing, IEEE Transactions on, vol. 59, no. 11, pp. 5577-5588, Nov 2011.

[14] E. Masazade, R. Niu, and P. Varshney, "Dynamic bandwidth allocation for target tracking in wireless sensor networks," in Information Fusion (FUSION), 2011 Proceedings of the 14th International Conference on, July 2011, pp. 1-8.

[15] _ - "Dynamic bit allocation for object tracking in wireless sensor networks," Signal Processing, IEEE Transactions on, vol. 60, no. 10, pp. 5048-5063, Oct 2012.

[16] A. Ababneh, L. Akter, and F. Zghoul, "Quantizer design for RSSI-based target localization in sensor networks."

[17] Y. Zhang, C. Lee, D. Niyato, and P. Wang, "Auction approaches for resource allocation in wireless systems: A survey," IEEE Communications Surveys Tutorials, vol. 15, no. 3, pp. 1020-1041, Third 2013.

[18] S. Sengupta and M. Chatterjee, "Designing auction mechanisms for dynamic spectrum access," Mobile Networks and Applications, vol. 13, no. 5, pp. 498-515, 2008.

[19] J. Sun, E. Modiano, and L. Zheng, "Wireless channel allocation using an auction algorithm," Selected Areas in Communications, IEEE Journal on, vol. 24, no. 5, p. 1085, 2006.

[20] J. Bae, E. Beigman, R. A. Berry, M. L. Honig, and R. Vohra, "Sequential bandwidth and power auctions for distributed spectrum sharing," Selected Areas in Communications, IEEE Journal on, vol. 26, no. 7, pp. 1193-1203, 2008.

[21] G. Wang, G. Cao, and T. LaPorta, "A bidding protocol for deploying mobile sensors," in Network Protocols, 2003. Proceedings. 11th IEEE International Conference on. IEEE, 2003, pp. 315-324.

[22] E. Elnahrawy, X. Li, and R. P. Martin, "The limits of localization using signal strength: A comparative study," in Sensor and Ad Hoc Communications and Networks, 2004. IEEE SECON 2004. 2004 First Annual IEEE Communications Society Conference on. IEEE, 2004, pp. 406-414.

[23] F. Gustafsson, F. Gunnarsson, and D. Lindgren, "Sensor models and localization algorithms for sensor networks based on received signal strength," EURASIP Journal on Wireless Communications and Networking, vol. 2012, no. 1, pp. 1-13, 2012.

[24] R. Niu and P. Varshney, "Target location estimation in sensor networks with quantized data," Signal Processing, IEEE Transactions on, vol. 54, no. 12, pp. 4519-4528, 2006.

[25] O. Ozdemir, R. Niu, and P. Varshney, "Channel aware target localization with quantized data in wireless sensor networks," Signal Processing, IEEE Transactions on, vol. 57, no. 3, pp. 1190-1202, 2009.

[26] H. V. Poor, An introduction to signal detection and estimation. Springer-Verlag, 1988.

[27] B. C. Levy, Principles of signal detection and parameter estimation. Springer, 2008

[28] W. R. Heinzelman, A. Chandrakasan, and H. Balakrishnan, "Energyefficient communication protocol for wireless microsensor networks," in System Sciences, 2000. Proceedings of the 33rd Annual Hawaii International Conference on, Jan 2000, pp. 10 pp. vol.2-.

[29] A. A. Abbasi and M. Younis, "A survey on clustering algorithms for wireless sensor networks," Computer Communications, vol. 30, no. 14, pp. 2826-2841, 2007.

[30] P. Saini and A. K. Sharma, "E-DEEC- enhanced distributed energy efficient clustering scheme for heterogeneous wsn," in Parallel Distributed and Grid Computing (PDGC), 2010 1st International Conference on, Oct 2010, pp. 205-210. 